\title{
$U(1)$ symmetry breaking in one-dimensional Mott insulators studied by the density matrix renormalization group method
}

\author{
Isao Maruyama, ${ }^{1}$ Tetsuji Koide, ${ }^{1}$ and Yasuhiro Hatsugai ${ }^{1,2}$ \\ ${ }^{1}$ Department of Applied Physics, University of Tokyo, 7-3-1 Hongo, Bunkyo-ku, Tokyo 113-8656, Japan \\ ${ }^{2}$ Institute of Physics, University of Tsukuba, 1-1-1 Tennodai, Tsukuba, Ibaraki 305-8571, Japan \\ (Received 13 October 2006; revised manuscript received 4 January 2007; published 6 December 2007)
}

A new type of external field violating the particle number preservation is studied in one-dimensional strongly correlated systems by the density matrix renormalization group method. Due to the $U(1)$ symmetry breaking, the ground state has fluctuation of the total particle number, which implies injection of electrons and holes from out of the chain. This charge fluctuation can be relevant even at half filling because the particle-hole symmetry is preserved under the finite effective field. In addition, we discuss a quantum phase transition obtained by considering the symmetry-breaking field as a mean field of interchain hopping.

DOI: 10.1103/PhysRevB.76.235105

PACS number(s): 71.10.Fd, 71.30.+h, 75.10.Lp

\section{INTRODUCTION}

A doped Mott insulator is one of possible candidates for superconductors with electron-electron correlation. At a rational filling, strong electron-electron interaction makes electrons localized in real space. This is the Mott insulator where charge excitations are gapped. Even at this rational filling, the spin degree of freedom survives as a gapless mode, where a quantum object as collection of the $S=1 / 2$ spins forms a singlet ground state.

When mobile carriers are introduced into the Mott insulator, we may expect that the charge gap is destroyed, which realizes a superconducting ground state driven by the electron interaction. In the resonating valence bond picture proposed by Anderson to describe high- $T_{c}$ superconducting cuprates, ${ }^{1,2}$ doped holes itinerate in a spin-singlet ground state and condense into a superconducting state. If there is no doped hole, i.e., the half-filled case, the spin-singlet ground state is expected as a Mott insulator.

Apart from real doping, that is, changing chemical potential, there can be several possibilities for effective carrier doping. One of a natural possibility can be geometrical frustration in layered organic superconductors, which is approximately described by a half-filled Hubbard model with nextnearest-neighbor hopping. ${ }^{3}$ Another is gossamer superconductivity proposed by Laughlin. ${ }^{4}$ Even at the halffilled case, finite double occupancy may destroy the Mott insulator at small on-site Coulomb repulsion and lead the ground state to the gossamer superconducting state., ${ }^{5,6}$ This theory has been also applied to organic superconductors. ${ }^{7}$ The gossamer superconducting state may not be realized as a realistic ground state of a typical strongly correlated system but is a good variational function based on a BardeenCooper-Schrieffer (BCS) superconducting state. However, it becomes an exact ground state of the model Hamiltonian ${ }^{4}$ which violates the charge conservation as the BCS Hamiltonian. It is theoretically interesting to consider $U(1)$ symmetry breaking generically.

Let us recall the BCS Hamiltonian here. The Hamiltonian with quadratic terms $\Delta_{k} c_{k \uparrow}^{\dagger} c_{-k \downarrow}^{\dagger}$ has been widely accepted as a theoretical model for superconductors, where $\Delta_{k}$ is a mean field of pair annihilation amplitude $\left\langle c_{k \uparrow} c_{-k \downarrow}\right\rangle$ and $c_{k \sigma}$ are an- nihilation operators of fermions. The mean field violates the $U(1)$ gauge symmetry, i.e., the total particle number is not preserved but fluctuating. This charge fluctuation turns out to diverge in the thermodynamic limit. ${ }^{8}$ The simplest candidate of $U(1)$ symmetry-breaking terms is $\Delta_{k} c_{k}^{\dagger}+$ H.c., which implies injection of electrons and holes from out of the system. The concept of this term is directly connected to local charge fluctuation or doping. Although the previous study is limited to the free fermion case, such a one-dimensional (1D) system defined as $H=\Sigma_{k} k c_{k}^{\dagger} c_{k}+\Delta \Sigma_{k}\left(c_{k}^{\dagger}+c_{k}\right)$ has been studied and was solved with a Jordan-Wigner transformation ${ }^{9}$ and with a canonical transformation. ${ }^{10}$ In Refs. 9 and 10, $\Delta$ terms were introduced in different contexts. It is common that the Hamiltonian is an effective one after tracing out of some environment. To clarify the meaning of $\Delta$, let us describe the procedure of Ref. 9 in detail; this spinless fermion chain can be mapped to semi-infinite $X Y$ model with a local magnetic field at the boundary. This local magnetic field in the $x y$ plane violates the number conservation of up (or down) spins and turns out to be $\Delta$. In addition, the injection of electrons by this $U(1)$ symmetry-breaking term has been studied in one-dimensional Josephson junction arrays. ${ }^{11}$

There are two motivations of the present work. The first is to clarify the properties of strongly correlated systems under a new type of external field: $\Delta_{i \sigma} c_{i \sigma}^{\dagger}+$ H.c., where $\Delta_{i \sigma}$ depends on the site $i$ and spin $\sigma$. Especially, the particle-hole symmetric Hubbard model with $\Delta_{i \sigma}$ has the ground state which is a superposition of electron-doped states, hole-doped states, and the half-filled Mott insulating state, which evokes the gossamer theory. It is interesting to evaluate fluctuation of total particle number for an incompressible Mott insulator as a direct measure of the $U(1)$ symmetry breaking. The other is to test a mean-field-type approach for the interchain hopping of fermion chains. When we consider a decoupling of interchain hopping $t_{\perp} c_{i \sigma}^{\dagger} c_{\perp i \sigma}$ into $\Delta_{i \sigma} c_{i \sigma}^{\dagger}$ with the mean-field-type approximation, the effective fields $\Delta_{i \sigma}$ can be identified as $\left\langle t_{\perp} c_{i \sigma}\right\rangle \cdot{ }^{24}$ In this approach, the Hamiltonian is considered as an effective one obtained after tracing out neighboring chains in the quasi-one-dimensional (quasi-1D) systems. In this sense, this mean field induces fluctuation of total charge of each chain due to the interchain hopping.

Since the Hubbard model is a strongly correlated system, we adopt the density matrix renormalized group (DMRG) 
method, which is one of powerful numerical methods for 1D quantum systems. ${ }^{12-14}$ The method is quite accurate in various 1D systems, while application to two-dimensional systems is difficult. As an application to higher dimensions, we note that the DMRG method has been already used in quasi-1D spin systems with interchain couplings treated as mean fields. ${ }^{15}$ The present paper is an attempt to quasi-1D fermionic systems with interchain hopping as a mean field.

The paper is organized as follows. In Sec. II, we construct a Hamiltonian with a generalized $U(1)$ breaking term and mention a "bath" site introduced by the canonical transformation. ${ }^{10}$ In Sec. III, we describe an application of the DMRG method to the Hamiltonian which does not conserve particle number. In Sec. IV, we demonstrate the meanfield-type approach for quasi-1D strongly correlated electron systems. Finally, we conclude the paper with a discussion of the mean-field-type approach. In the Appendix, the note for the canonical transformation is given.

\section{CORRELATED ELECTRON SYSTEMS WITH $U(1)$ SYMMETRY-BREAKING TERM}

Let us define a Hamiltonian with the generalized symmetry-breaking term $H_{\Delta}$,

$$
H=H_{0}+H_{\Delta},
$$

where $H_{0}$ can be any Hamiltonian for a correlated electrons system. In this paper, we restrict $H_{0}$ to the Hubbard model defined as

$$
H_{0}=-t \sum_{i=1}^{L-1} \sum_{\sigma} c_{i+1, \sigma}^{\dagger} c_{i \sigma}+\text { H.c. }+U \sum_{i=1}^{L}\left(n_{i \uparrow}-\frac{1}{2}\right)\left(n_{i \downarrow}-\frac{1}{2}\right),
$$

where $c_{i \sigma}$ is a fermion operator and $L$ is the system size. The symmetry-breaking term $H_{\Delta}$ is defined as

$$
H_{\Delta}=\sum_{i \sigma} \Delta_{i \sigma}^{*} c_{i \sigma}+\Delta_{i \sigma} c_{i \sigma}^{\dagger},
$$

where $\Delta_{i \sigma}$ are considered as external fields at this stage. This model with nonzero $\Delta_{i \sigma}$ breaks the particle number conservation, because $H_{\Delta}$ is not commutable with the total number of particles, $\left[H_{\Delta}, N_{\text {tot }}\right] \neq 0$, where $N_{\text {tot }}=\Sigma c_{i \sigma}^{\dagger} c_{i \sigma}$. This is due to the fact that $H_{\Delta}$ breaks the $U(1)$ symmetry, where the global $U(1)$ rotation is defined as $c_{i \sigma} \rightarrow e^{i \theta} c_{i \sigma}$.

Generally, an external field breaks some symmetry as a magnetic field breaks a spin rotational symmetry. In addition to the $U(1)$ symmetry breaking, $H_{\Delta}$ also breaks the $S U(2)$ spin-rotational symmetry, while $H_{0}$ is an $S U(2)$ invariant, where the global $S U(2)$ rotation is defined as $\boldsymbol{c}_{i} \rightarrow U \boldsymbol{c}_{i}$, where $\boldsymbol{c}_{i}==^{t}\left(c_{i \uparrow}, c_{i \downarrow}\right)$ and $\operatorname{det} U=1$. The symmetry-breaking term transforms under the $S U(2)$ rotation as

$$
\begin{gathered}
H_{\Delta}\left(\boldsymbol{\Delta}_{i}\right)=\sum_{i} \boldsymbol{c}_{i}^{\dagger} \boldsymbol{\Delta}_{i}+\boldsymbol{\Delta}_{i}^{\dagger} \boldsymbol{c}_{i} \\
\rightarrow H_{\Delta}^{\prime}=H_{\Delta}\left(U^{\dagger} \boldsymbol{\Delta}_{i}\right),
\end{gathered}
$$

where $\boldsymbol{\Delta}_{i}={ }^{t}\left(\Delta_{i \uparrow}, \Delta_{i \downarrow}\right)$. The symmetry is recovered only if $\Delta_{i \sigma}=0$ for all $i, \sigma$.

\section{A. Particle-hole symmetry}

Let us suppose that $H_{0}$ satisfies the particle-hole symmetry, i.e., $H_{0}$ is invariant under the usual particle-hole transformation on the tight-binding model: $c_{i \sigma} \rightarrow(-1)^{i} c_{i \sigma}^{\dagger}$. In other words, $H_{0}$ commutes with an antiunitary operator $\Theta,{ }^{16}$ defined as $\Theta=K U_{\mathrm{ph}}$, where $K$ is a complex conjugation and $U_{\mathrm{ph}}$ is the unitary operator defined as $U_{\mathrm{ph}}=\mathbf{i}^{L} \prod_{i \sigma}\left[c_{i \sigma}+(-1)^{i} c_{i \sigma}^{\dagger}\right]$. It satisfies

$$
\Theta^{-1} c_{i \sigma} \Theta=U_{\mathrm{ph}}^{-1} c_{i \sigma} U_{\mathrm{ph}}=(-1)^{i} c_{i}^{\dagger},
$$

and one can show

$$
\begin{gathered}
\Theta^{-1} H_{0} \Theta=H_{0}, \\
\Theta^{-1} H_{\Delta}\left(\boldsymbol{\Delta}_{i}\right) \Theta=H_{\Delta}\left((-1)^{i} \boldsymbol{\Delta}_{i}\right),
\end{gathered}
$$

where $t$ can be complex. We take $t=1$ as a unit of energy in the numerical calculations. The symmetry-breaking term $H_{\Delta}$ with $\boldsymbol{\Delta}_{i}=(-1)^{i} \boldsymbol{\Delta}_{i}$ is also invariant under the particle-hole transformation, where the Hamiltonian $H=H_{0}+H_{\Delta}$ preserves the particle-hole symmetry but breaks $U(1)$ and $S U(2)$ symmetries. It is easy to show that the total number of electrons of this Hamiltonian is half filled when the ground state is unique. The proof is as follows: Because of the particle-hole symmetry $\Theta^{-1} H \Theta=H, \Theta^{-1}|\mathrm{gs}\rangle$ is also the ground state, $H \Theta^{-1}|\mathrm{gs}\rangle=E_{g s} \Theta^{-1}|\mathrm{gs}\rangle$. Since the ground state is unique, $|\mathrm{gs}\rangle$ is proportional to $\Theta^{-1}|\mathrm{gs}\rangle$ except for a phase factor. Then, one obtains

$$
\left\langle\mathrm{gs}\left|N_{\text {tot }}\right| \mathrm{gs}\right\rangle=\left\langle\mathrm{gs}\left|\Theta^{-1} N_{\text {tot }} \Theta\right| \mathrm{gs}\right\rangle,
$$

and the total number $N_{\text {tot }}$ satisfies

$$
\Theta^{-1} N_{\text {tot }} \Theta=2 L-N_{\text {tot }} \text {. }
$$

From Eqs. (4) and (5), it is deduced that total number of electrons is half filled, $\left\langle N_{\text {tot }}\right\rangle=L$. It might be interesting to remind the reader that the half-filled Hamiltonian with the $U(1)$ symmetry-breaking term has some analogy to the halffilled case of the gossamer superconducting theory with a finite double occupancy.

\section{B. Hidden even-odd parity conservation}

To handle the fermion sign by the DMRG method, let us consider the following extension of the Hilbert space by the canonical transformation: ${ }^{10}$

$$
c_{i \sigma} \rightarrow \tilde{c}_{i \sigma}=\left(\alpha+\alpha^{\dagger}\right) c_{i \sigma},
$$

where $\alpha$ is an additional annihilation operator of a spinless fermion and satisfies

$$
\left\{\alpha, c_{i \sigma}\right\}=0, \quad\left\{\alpha^{\dagger}, c_{i \sigma}\right\}=0 .
$$

Anticommutation relations of $\tilde{c}_{i \sigma}$ are easily shown as $\left\{\widetilde{c}_{i \sigma}, \widetilde{c}_{i^{\prime} \sigma^{\prime}}\right\}=-\left\{c_{i \sigma}, c_{i^{\prime} \sigma^{\prime}}\right\}=0 \quad$ and $\quad\left\{\widetilde{c}_{i \sigma}, \widetilde{c}_{i^{\prime} \sigma^{\prime}}^{\dagger}\right\}=\left\{c_{i \sigma}, c_{i^{\prime} \sigma^{\prime}}^{\dagger}\right\}$ $=\delta_{i i^{\prime}} \delta_{\sigma \sigma^{\prime}}$. Moreover, $H_{0}$ is invariant under this transformation,

$$
H_{0}=H_{0}\left(\left\{c_{i \sigma}\right\}\right) \rightarrow \widetilde{H_{0}}=H_{0}\left(\left\{\widetilde{c}_{i \sigma}\right\}\right)=H_{0}\left(\left\{c_{i \sigma}\right\}\right),
$$

because $H_{0}$ is made of invariant operators as $\widetilde{c}_{i \sigma^{\prime}}^{\dagger} \widetilde{c}_{i^{\prime} \sigma^{\prime}}$ $=c_{i \sigma}^{\dagger} c_{i^{\prime} \sigma^{\prime}}$. However, the term $\widetilde{H}_{\Delta}$ is modified as 


$$
H_{\Delta} \rightarrow \widetilde{H_{\Delta}}=\sum_{i \sigma} \Delta_{i \sigma}^{*}\left(\alpha+\alpha^{\dagger}\right) c_{i \sigma}+\Delta_{i \sigma} c_{i \sigma}^{\dagger}\left(\alpha+\alpha^{\dagger}\right)
$$

This implies that $\alpha$ site is a "environment bath" site in the spirit of the dynamical mean field theory. ${ }^{17}$ The operator of the total particle number $N_{\text {tot }}$ is invariant under the transformation, i.e., $\tilde{N}_{\text {tot }}=N_{\text {tot }}$. The total Hamiltonian $\widetilde{H}$ conserves neither $\tilde{N}_{\text {tot }}$ nor the total particle number including $\alpha, \tilde{N}_{\alpha}$ $=\tilde{N}_{\text {tot }}+\alpha^{\dagger} \alpha$. However, the parity of $\tilde{N}_{\alpha}$ is conserved.

The parity operator of $\widetilde{N}_{\alpha}$ is defined as

$$
\widetilde{P}=e^{\mathbf{i} \pi \tilde{N}_{\alpha}}=e^{\mathbf{i} \pi \alpha^{\dagger} \alpha} \prod_{i \sigma} e^{\mathbf{i} \pi c_{i \sigma}^{\dagger} c_{i \sigma}}
$$

and satisfies $\widetilde{P}^{\dagger} \widetilde{P}=1$ and $\widetilde{P}=\widetilde{P}^{\dagger}$. Since $\widetilde{H}$ is bilinear, one can show that the even-odd parity of $\widetilde{N}_{\alpha}$ is conserved, i.e.,

$$
[\tilde{H}, \tilde{P}]=0 .
$$

Since $\widetilde{P}$ is conserved, one can take simultaneous eigenstates of $\widetilde{H}$ and $\widetilde{P}$ as

$$
\begin{gathered}
\tilde{H}|\widetilde{\Psi}(E, p)\rangle=E|\widetilde{\Psi}(E, p)\rangle, \\
\widetilde{P}|\widetilde{\Psi}(E, p)\rangle=p|\widetilde{\Psi}(E, p)\rangle,
\end{gathered}
$$

where $E$ is eigenenergy and $p$ is \pm 1 . The ground states $|\widetilde{\Psi}(E, p)\rangle$ for $\widetilde{H}$ are doubly degenerated if the ground state $|\Psi(E)\rangle$ for the original Hamiltonian $H$ is unique. As shown in the Appendix, one can show that expectation value of any operator $\mathcal{O}$ in the original Fock space is written as $\langle\Psi(E)|\mathcal{O}| \Psi(E)\rangle=\langle\widetilde{\Psi}(E ;+)|\widetilde{\mathcal{O}}| \widetilde{\Psi}(E ;+)\rangle=\langle\widetilde{\Psi}(E ;-)|\widetilde{\mathcal{O}}| \widetilde{\Psi}(E$ $-)\rangle$. This means that any expectation value for the original system $H$ can be obtained from the system $\widetilde{H}$. We note that such operators with $\alpha$ as $\widetilde{N}_{\alpha}$ has no corresponding operator in the original Fock space.

\section{METHOD}

To study the 1D strongly correlated system without conservation of the total particle number, we use the DMRG method. As we implied in the previous section, the conservation of even-odd parity of particle number is required to handle the fermion sign in the DMRG algorithm. In this section, we will illustrate the algorithm in detail. We note that the DMRG method has been applied to the different model which does not conserve the total number of particles but conserves its parity, for example, a $t-J$ model with a BCS mean field ${ }^{18}$ or the BCS pairing Hamiltonian. ${ }^{19}$

First of all, we describe the iterative procedure of the DMRG for the Hamiltonian $\widetilde{H}$ with $\alpha$ site. Figure 1 illustrates a system in the first iterative procedure enlarging the system size from $L=4$ to $L=6$. As seen in Fig. 1(b), the hopping terms between $\alpha$ site and each sites in $\widetilde{H}_{\Delta}$ become long range in the successive elongation. Generally speaking, long-range hopping terms such as $c_{1}^{\dagger} c_{L}$ increase numerical errors, but the present situation is better because the $\alpha$ site is not renormalized in this iterative procedure. (a)
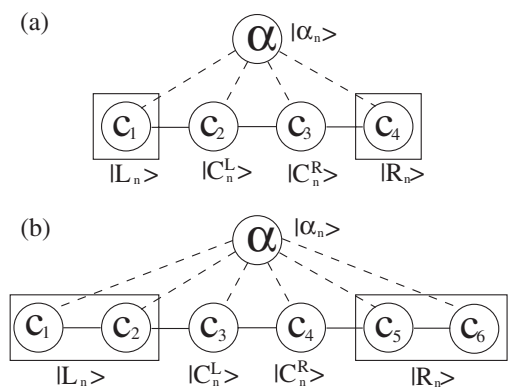

FIG. 1. The system in the first step for infinite system algorithm of the DMRG with the bath site $\alpha$. The system size is enlarged from (a) $L=4$ to (b) $L=6$. The $\alpha$ site, left block, center-left site, centerright site, and right block are represented by indices $\left|\alpha_{n}\right\rangle,\left|L_{n}\right\rangle,\left|C_{n}^{L}\right\rangle$, $\left|C_{n}^{R}\right\rangle$, and $\left|R_{n}\right\rangle$.

Next, to handle a fermionic system, one needs to deal with the fermion sign. ${ }^{13}$ When the two local operators $\hat{A}$ and $\hat{B}$ are represented in bases $\left|A_{n}\right\rangle$ and $\left|B_{n}\right\rangle$, one can get the product as $\left\langle A_{n} B_{m}|\hat{A} \hat{B}| A_{n^{\prime}} B_{m^{\prime}}\right\rangle= \pm\left\langle A_{n}|\hat{A}| A_{n^{\prime}}\right\rangle\left\langle B_{m}|\hat{B}| B_{m^{\prime}}\right\rangle$, where the signature \pm comes from the fermion sign. This formula is valid if the states $\left|A_{n}\right\rangle$ and $\left|B_{n}\right\rangle$ have a fixed evenodd parity of particle number and operators $\hat{A}$ and $\hat{B}$ conserve the even-odd parity. Otherwise, states need to be modified by the rearrangement; for example, such states as (1 $\left.+c^{\dagger}\right)|0\rangle$ may change to $\left(1-c^{\dagger}\right)|0\rangle$, which is not an easy task for the DMRG procedure. This is the reason why conservation of even-odd parity is required by the DMRG.

The canonical transformation makes it possible to calculate the fermion sign because all operators conserve the even-odd parity as shown in Eq. (A1) in the Appendix. Moreover, local bases $\left|L_{n}\right\rangle,\left|C_{n}^{L}\right\rangle,\left|C_{n}^{R}\right\rangle,\left|R_{n}\right\rangle$, and $\left|\alpha_{n}\right\rangle$ are eigenstates of the parity operator. To explain it, let us describe one step of the iterative procedure below.

(1) Here, we suppose that local bases have fixed parity of particle number as in $L=4$ system.

(2) Make the matrix elements of the Hamiltonian $\widetilde{H}$ from local operators represented by local bases, taking care of the fermion sign. The Hamiltonian $\widetilde{H}$ is block diagonalized into even and odd parity sectors.

(3) Calculate the ground states $\left|\widetilde{\Psi}\left(E_{g s}, p\right)\right\rangle$ and its energy $E_{g s}$, where the even-odd parity of particle number is denoted by $p= \pm$.

(4) Make the matrix elements of four density matrices $\rho_{L / R, \pm}$,

$$
\rho_{L, \pm}=\underset{R, C^{R}, \alpha}{\operatorname{Tr}} \rho_{ \pm}, \quad \rho_{R, \pm}=\underset{L, C^{L}, \alpha}{\operatorname{Tr}} \rho_{ \pm},
$$

where $\rho_{ \pm}=\left|\widetilde{\Psi}\left(E_{g s}, \pm\right)\right\rangle\left\langle\widetilde{\Psi}\left(E_{g s}, \pm\right)\right|$. One can show that these density matrices are block diagonalized into even and odd parity sectors, i.e., $\left[\rho_{L / R, \pm}, e^{\left.\mathbf{i} \pi N_{L / R}\right]}=0\right.$, as the Hamiltonian is.

(5) Diagonalize $\rho_{L / R, \pm}$ and select the lowest $m$ eigenvalues and their eigenvectors called renormalized bases, which are eigenstates of $e^{\mathbf{i} \pi N_{L / R}}$.

(6) Remake matrix elements of all local operators in the renormalized bases. Then, renormalized bases are the next 
local bases and satisfy the supposition in the step (1).

As described in the procedure, since $\tilde{H}$ conserves evenodd parity, one can show that each local base in each steps has fixed parity of particle number. That is, one can calculate the fermion sign.

Finally, we note that the number of states of left and right blocks, $m$, is used up to about 60 to obtain the truncation error of less than $10^{-4}$. Since we deal with general $\Delta_{i \sigma}$ which depends on the spatial site, the DMRG method for the random system ${ }^{20}$ is employed. Although we implemented the infinite-size and finite-size methods, we found that the improvement of the accuracy due to the finite-size method is small for $L \leqslant 40$ and $m=64$. One reason is that the DMRG for random systems has more information about the boundary than usual DMRG.

The expectation value is evaluated as

$$
\langle A\rangle=\frac{1}{2} \sum_{p= \pm}\left\langle\widetilde{\Psi}\left(E_{g s}, p\right)|\widetilde{A}| \widetilde{\Psi}\left(E_{g s}, p\right)\right\rangle
$$

to avoid the numerical error. We note that we restrict the system size $L$ to even to obtain the unique ground state $|\Psi(E)\rangle$ for $H$. In the DMRG calculation, $\tilde{H}$ is used, where the ground states $|\widetilde{\Psi}(E, p)\rangle$ for $\tilde{H}$ are doubly degenerated after the canonical transformation.

\section{RESULTS}

In the following, we will show numerical evaluation of total-charge fluctuation in Sec. IV A, which is the direct consequence of external fields $\Delta_{i \sigma}$. In Sec. IV B, we will show a demonstration of the mean-field theory to deal with the interchain hopping.

\section{A. Total-charge fluctuation}

The ground state for $H_{0}$ has a fixed number of electrons because the Hamiltonian $H_{0}$ is commutable with $\hat{N}$, where the total-charge fluctuation, $\Delta N=\sqrt{\left\langle\hat{N}^{2}\right\rangle-\langle\hat{N}\rangle^{2}}$, is zero when the ground state is unique. On the other hand, since the external field $\Delta_{i \sigma}$ breaks the $U(1)$ symmetry, the total-charge fluctuation becomes finite. The ground state for nonzero $\Delta_{i \sigma}$ is a superposition of electron-doped and hole-doped states. It implies that, even at half filling, "effective carriers" are introduced by the nonzero $\Delta_{i \sigma}$.

In Fig. 2, total-charge fluctuation $\Delta N$ as a function of $1 / U$ is plotted. When the Coulomb interaction $U$ becomes infinite, doped states are not allowed at half filling and $\Delta N$ becomes zero. Finite total-charge fluctuation is proportional to $1 / U$. This means that large charge gap prefers no fluctuation. It should be noted that in large $1 / U$ region, total-charge fluctuation $\Delta N$ is of order of unity, $\Delta N \sim \mathcal{O}(1)$. That is, the present model cannot reproduce the BCS ground state with $\Delta N \sim \mathcal{O}\left(L^{1 / 2}\right)$. Since the charge compressibility is defined as $\kappa(\mu)=\frac{\partial n}{\partial \mu}=\frac{1}{L} \frac{\partial\left\langle N_{\text {tot }}\right\rangle}{\partial \mu}$, the charge compressibility is expected to be zero in the limit $L \rightarrow \infty$ at half filling even in our model with finite $\Delta$.

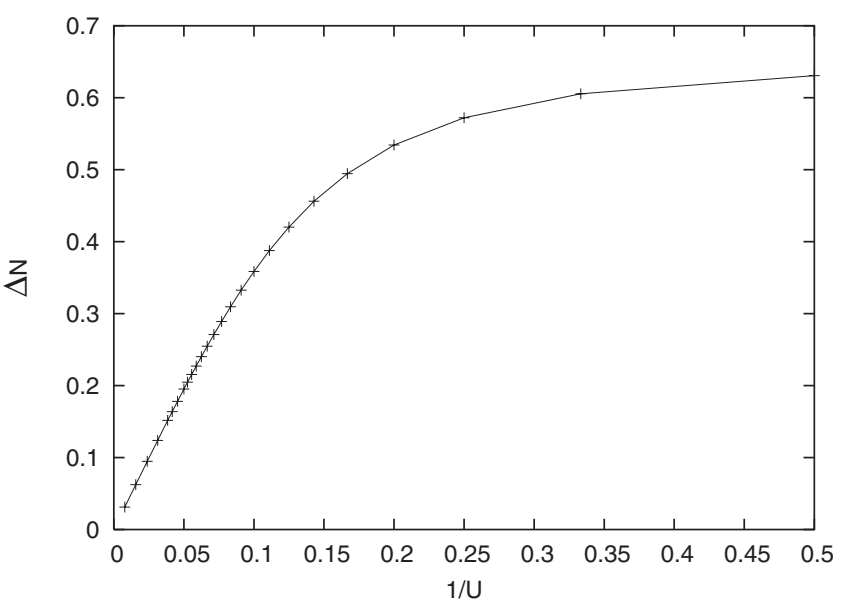

FIG. 2. An example of total-charge fluctuation $\Delta N$ as a function of $1 / U$ for system-size $L=4$ at half filling with the Hamiltonian $H=H_{0}+H_{\Delta}$ at $t=1$ and particle-hole symmetric $\Delta_{n \sigma}=\cos (n \pi / 4)$, where $\left\langle N_{\text {tot }}\right\rangle=L$ is satisfied numerically. This figure is only for the small system and relatively large $\Delta_{i \sigma}$, but the qualitative character that $\Delta N$ is proportional to $1 / U$ is general.

\section{B. Interchain hopping as mean fields}

In this section, let us consider the interchain hopping of 1D Mott insulators. When we take an ansatz of the meanfield type, the external field $\Delta_{i \sigma}$ can be determined selfconsistently as $-t_{\perp} c_{i \sigma}^{\dagger} c_{i_{\perp} \sigma} \sim c_{i \sigma}^{\dagger} \Delta_{i \sigma}$. We adopt the selfconsistent equation

$$
\Delta_{i \sigma}=-t_{\perp}\left\langle c_{i \sigma}\right\rangle .
$$

Although the interchain hopping also gives rise to effects of the band structure and the dimensionality, they are not taken into account in the present approach. The meaning of $t_{\perp}$ in the self-consistent equation is the strength of charge fluctuation in the perpendicular direction with the general band structure. In other words, $t_{\perp}$ is the hopping energy between the 1D system and the surrounding environment in analogy with Refs. 9 and 10, where the Hamiltonian is an effective one after tracing out of some environment.

In the DMRG method, we used the transformed Hamiltonian $\tilde{H}$ and the transformed self-consistent equation as $\Delta_{i \sigma}=-t_{\perp}\left\langle\left(\alpha+\alpha^{\dagger}\right) c_{i \sigma}\right\rangle$. We note that $\Delta_{i \sigma}$ is limited to real number and left-right symmetric for simplicity. In Fig. 3, some results of converged $\Delta_{i \sigma}$ after the self-consistent loop for $L=20$ are plotted. Converged $\Delta_{i \sigma}$ decreases as the interaction $U$ increases. Also, we have observed a quantum phase transition from nonzero $\Delta_{i \sigma}$ to zero $\Delta_{i \sigma}$.

To clarify the transition, we define the stabilization energy $\Delta E=E(0)-E\left(\Delta_{i \sigma}\right)$, which implies the energy gain due to the charge fluctuation in the perpendicular direction, where $E\left(\Delta_{i \sigma}\right)$ is the ground state energy with converged $\Delta_{i \sigma}$. There are two simple limits: infinite $U$ limit and small $t_{\perp}$ limit. In both cases, $\Delta_{i \sigma}$ converged to about zero and the $1 \mathrm{D}$ Mott insulator is realized. In Fig. 4, the stabilization energy is plotted as a function of $t_{\perp}$. Increasing $t_{\perp}$ means that the length between chains is changing more closely, which corresponds to applying pressure. In the small $t_{\perp}$ region, $\Delta E$ 


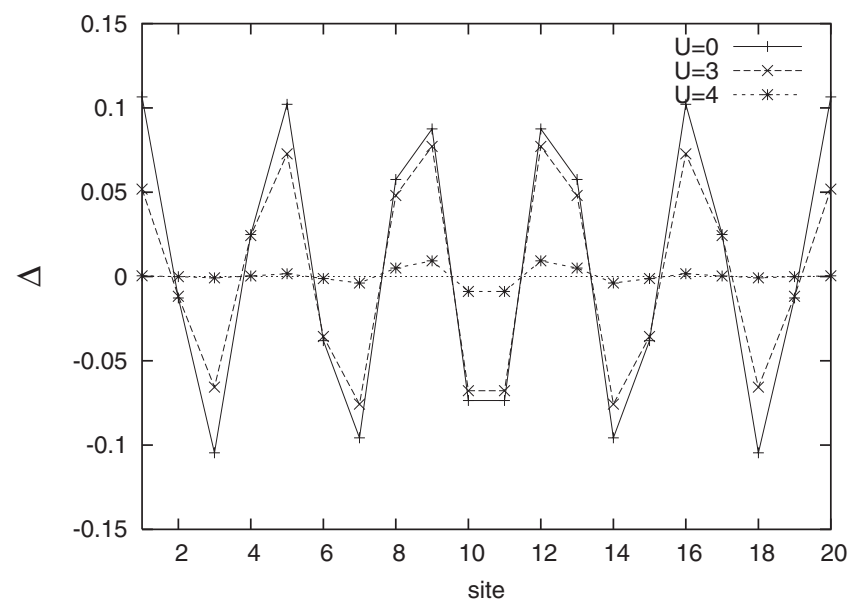

FIG. 3. Converged $\Delta_{i \sigma}=\Delta_{i}$ as a real function of site $i$ with varying $U=0,3,4$. The system size is $L=20$.

becomes zero, which is identified as the 1D Mott insulator phase. Actually, converged $\Delta_{i \sigma}$ and $\Delta N$ are zero there. There is a transition from the 1D Mott insulator phase to the symmetry-breaking phase as $t_{\perp}$ increase. Extrapolated values in Fig. 4 are consistent with a critical point $t_{\perp}^{c} / t=0.16$ for $U / t=2$. Since the charge gap of Mott chain of $U / t=2$ is $\Delta_{c}$ $=0.17,{ }^{21}$ the naive criterion $t_{\perp}^{c} \sim \Delta_{c}$ (Ref. 22) is reasonable in this analysis.

\section{CONCLUSION}

In conclusion, we have numerically studied effects of $U(1)$ and $S U(2)$ symmetry breakings due to the external fields $\Delta_{i \sigma}$, which lead to nonzero total-charge fluctuation $\Delta N$. Finite $\Delta N$ means that the ground state is a superposition of electron-doped and hole-doped states. We have applied the DMRG method to the particle-hole symmetric Hubbard chain with $\Delta_{i \sigma}$ and demonstrated that the total-charge fluctuation $\Delta N$ at zero temperature is linear in $1 / U$ even at halffilled case.

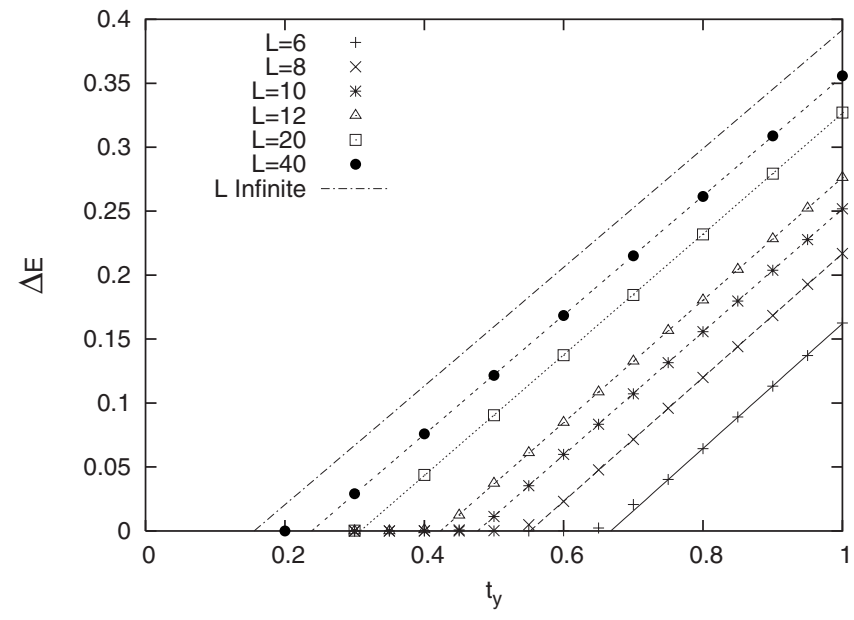

FIG. 4. Stabilization energy $\Delta E=E(0)-E\left(\Delta_{i \sigma}\right)$ as a function of $t_{\perp}$ with converged $\Delta_{i \sigma}$ for $U / t=2$. Points are obtained by the DMRG, and lines are fitted with a linear function.
Considering $\Delta_{i \sigma}$ as a mean field of interchain hopping tentatively, we have obtained the quantum phase transition from the 1D Mott insulator to the symmetry-breaking phase as $t_{\perp}$ increases. The transition point $t_{\perp}^{c}$ in Fig. 4 is in reasonable agreement with the naive criterion. ${ }^{22}$ In the symmetrybreaking phase, effectively doped carriers itinerate between chains by the nonzero $\Delta_{i \sigma}$. Since the difference between two phases is whether the interchain hopping becomes relevant or not, one may say that this transition is a deconfinement transition. ${ }^{22}$

As described in Sec. IV, the magnitude of $\Delta N$ as a function of the system size is constant while the BCS theory gives $\Delta N \sim \mathcal{O}\left(L^{1 / 2}\right)$. This property may be related to the fact that we dropped the anticommutation relation between $\Delta_{i \sigma}$ and fermion operators in the Hamiltonian. That is, expectation value $\left\langle c_{i \sigma}\right\rangle$ was a fermionic operator before taking the average as a mean field. As pointed out in Ref. 23, this fact gives the limitation of this "mean field" approach. To bosonize the mean field will be published elsewhere.

Due to the limitation, the clarification of the symmetrybreaking phase remains a future work. The clarification is interesting, because it is well known that destroying the Mott phase by applying the pressure, i.e., increasing $t_{\perp}$, is typical for high- $T_{c}$ cuprates.

To deal with nonuniform $\Delta_{i \sigma}$, the DMRG method for random systems ${ }^{20}$ is used, and improvement due to the finitesize method from the infinite-size method was negligible in this paper. As a technical outlook, we can update the selfconsistent field $\Delta_{i \sigma}$ during the finite-size method. The combination of finite method and self-consistent loop will improve the cost of calculation time, where the self-consistent field is calculated at the center block in sweep of the finite method. In this method, DMRG is combined with the meanfield theory more closely.

\section{ACKNOWLEDGMENTS}

This work was in part supported by Grant-in-Aid for Scientific Research (No. 17540347) from JSPS and on Priority Areas (Grant No. 18043007) from MEXT. Y.H. was also supported in part by the Sumitomo Foundation. Some of the numerical calculations were carried out on Altix3700BX2 at YITP in Kyoto University.

\section{APPENDIX: CANONICAL TRANSFORMATION}

To describe properties of the canonical transformation, we introduce the Majorana fermions defined as

$$
\begin{gathered}
\alpha_{+}=\alpha+\alpha^{\dagger}, \\
\alpha_{-}=-\mathbf{i}\left(\alpha-\alpha^{\dagger}\right),
\end{gathered}
$$

which are unitary and Hermite and satisfy anticommutation relations $\left\{\alpha_{+}, \alpha_{-}\right\}=0$ and $\left\{\alpha_{ \pm}, c_{i \sigma}\right\}=0$. It can be proven that even parity of $\widetilde{P}$ defined in Eq. (6) is anticommutable with $\alpha_{-}:\left\{\tilde{P}, \alpha_{-}\right\}=0$. In addition, the canonical transformation maps any operator $\mathcal{O}$ in the original system into $\widetilde{\mathcal{O}}$, which satisfies 


$$
[\widetilde{P}, \widetilde{\mathcal{O}}]=\left[\alpha_{-}, \widetilde{\mathcal{O}}\right]=0
$$

because $\widetilde{\mathcal{O}}$ does not contain $\alpha_{-}$. Finally, since the transformation preserves the anticommutation relations, the new vacuum $\left(c_{i \sigma}|\widetilde{0}\rangle=\alpha|\widetilde{0}\rangle=0\right)$ satisfies

$$
\langle 0|\mathcal{O}| 0\rangle=\langle\widetilde{0}|\widetilde{\mathcal{O}}| \widetilde{0}\rangle .
$$

We note that the Fock space based on the new vacuum is enlarged from the original Fock space.

To define the states in the new Fock space, we write the bases in the original Fock space explicitly as

$$
|I\rangle:=\left|\left\{n_{i}\right\}\right\rangle=\prod_{i=1}^{2 L}\left(c_{i}^{\dagger}\right)^{n_{i}}|0\rangle .
$$

After the transformation, the bases are mapped into

$$
\left.\left.|\widetilde{I}\rangle:=\mid \widetilde{\left\{n_{i}\right.}\right\}\right\rangle=\prod_{i=1}^{2 L}\left(\alpha_{+} c_{i}^{\dagger}\right)^{n_{i}}|\widetilde{0}\rangle .
$$

Since $\alpha_{+}^{2}=1$, the bases have even parity of $\widetilde{P}: \widetilde{P}|\widetilde{I}\rangle=|\widetilde{I}\rangle$, where $\widetilde{P}$ is defined in Eq. (6). When we define $|\widetilde{I} ;+\rangle=|\widetilde{I}\rangle$ and $\mid \widetilde{I}$; $-\rangle=\alpha_{-}|\widetilde{I}\rangle$, one can easily show that a set of $2 \times 4^{L}$ bases $|\tilde{I} ; \pm\rangle$ is the orthonormalized complete set and the bases satisfy $\widetilde{P}|\widetilde{I} ; \pm\rangle= \pm|\widetilde{I} ; \pm\rangle$ because of $\left\{\widetilde{P}, \alpha_{-}\right\}=0$.
With Eq. (A1) and $\alpha_{-}^{2}=1$, one can show $\left\langle\widetilde{I} ;+|\widetilde{\mathcal{O}}| \widetilde{I^{\prime}} ;+\right\rangle$ $=\left\langle\widetilde{I} ;-|\widetilde{\mathcal{O}}| \widetilde{I^{\prime}} ;-\right\rangle$. With Eq. (A2), one can also show $\langle I|\mathcal{O}| I\rangle$ $=\left\langle\widetilde{I} ;+|\widetilde{\mathcal{O}}| \widetilde{I}^{\prime} ;+\right\rangle$. Combining them, we summarize

$$
\left\langle I|\mathcal{O}| I^{\prime}\right\rangle=\left\langle\widetilde{I} ;+\mid \widetilde{\mathcal{O} \mid I^{\prime}} ;+\right\rangle=\left\langle\widetilde{I} ;-\mid \widetilde{\mathcal{O} \mid I^{\prime}} ;-\right\rangle
$$

This means that the block diagonalized operator has the same matrix elements for even-odd sectors. Since the matrix elements of the Hamiltonian also satisfy Eq. (A5), the eigenvectors of $H$ and $\widetilde{H}$ can be written with the same elements $C_{I}(E)$,

$$
\begin{gathered}
|\Psi(E)\rangle=\sum_{I} C_{I}(E)|I\rangle, \\
|\widetilde{\Psi}(E, \pm)\rangle=\sum_{I} C_{I}(E)|\widetilde{I} ; \pm\rangle,
\end{gathered}
$$

where $C_{I}(E)$ satisfies the Schrödinger equation $\left\langle I|H| I^{\prime}\right\rangle C_{I^{\prime}}(E)=E C_{I}(E)$. We note that degenerated eigenvectors $|\widetilde{\Psi}(E, \pm)\rangle$ satisfy $\left\langle\widetilde{\Psi}(E, p) \mid \widetilde{\Psi}\left(E^{\prime}, p^{\prime}\right)\right\rangle=\delta_{E E^{\prime}} \delta_{p p^{\prime}}$.

We conclude that the expectation value for any operator $\mathcal{O}$ can be written as

$$
\begin{aligned}
\langle\Psi(E)|\mathcal{O}| \Psi(E)\rangle= & \langle\widetilde{\Psi}(E ;+)|\widetilde{\mathcal{O}}| \widetilde{\Psi}(E ;+)\rangle=\langle\widetilde{\Psi}(E ; \\
& -)|\widetilde{\mathcal{O}}| \widetilde{\Psi}(E ;-)\rangle .
\end{aligned}
$$

${ }^{1}$ P. Anderson, Science 235, 1196 (1987).

${ }^{2}$ G. Kotliar, Phys. Rev. B 37, 3664 (1988).

${ }^{3}$ H. Yokoyama, M. Ogata, and Y. Tanaka, J. Phys. Soc. Jpn. 75, 114706 (2006).

${ }^{4}$ R. B. Laughlin, Philos. Mag. 86, 1165 (2006).

${ }^{5}$ F. C. Zhang, Phys. Rev. Lett. 90, 207002 (2003).

${ }^{6}$ J. Y. Gan, F. C. Zhang, and Z. B. Su, Phys. Rev. B 71, 014508 (2005).

${ }^{7}$ J. Y. Gan, Y. Chen, Z. B. Su, and F. C. Zhang, Phys. Rev. Lett. 94, 067005 (2005).

${ }^{8}$ M. Tinkham, Introduction to Superconductivity (McGraw-Hill, New York, 1996).

${ }^{9}$ F. Guinea, Phys. Rev. B 32, 7518 (1985).

${ }^{10}$ K. A. Matveev, Phys. Rev. B 51, 1743 (1995).

${ }^{11}$ T. Kato, Phys. Rev. B 65, 132511 (2002).

${ }^{12}$ J. Dukelsky and S. Pittel, Rep. Prog. Phys. 67, 513 (2004).

${ }^{13}$ N. Shibata, J. Phys. A 36, 381 (2003).
${ }^{14}$ U. Schollwoeck, Rev. Mod. Phys. 77, 259 (2005).

${ }^{15}$ D. J. Scalapino, Y. Imry, and P. Pincus, Phys. Rev. B 11, 2042 (1975).

${ }^{16}$ Y. Hatsugai, J. Phys. Soc. Jpn. 75, 123601 (2006).

${ }^{17}$ A. Georges, G. Kotliar, W. Krauth, and M. Rozenberg, Rev. Mod. Phys. 68, 13 (1996).

${ }^{18}$ S. R. White and D. J. Scalapino, Phys. Rev. B 57, 3031 (1998).

${ }^{19}$ J. Dukelsky and G. Sierra, Phys. Rev. Lett. 83, 172 (1999).

${ }^{20}$ K. Hida, J. Phys. Soc. Jpn. 65, 895 (1996).

${ }^{21}$ E. H. Lieb and F. Y. Wu, Phys. Rev. Lett. 20, 1445 (1968).

${ }^{22}$ S. Biermann, A. Georges, A. Lichtenstein, and T. Giamarchi, Phys. Rev. Lett. 87, 276405 (2001).

${ }^{23}$ T. Giamarchi, Quantum Physics in One Dimension (Oxford University Press, Oxford, 2004).

${ }^{24}$ The limitation of this approach, for example, the replacement of a fermionic operator by $c$ number, is discussed in Sec. V. 